\title{
Sul ruolo dell'ente nel contrasto alla corruzione: un moderno Atlante
}

\author{
On the role of the entity in the fight \\ against corruption: a modern Atlas
}

Riccardo Roscini-Vitali ${ }^{1}$

Università degli Studi di Verona, Italia riccardo.roscinivitali@univr.it

https://orcid.org/0000-0002-8967-8429

\section{Vittore d'Acquarone ${ }^{2}$}

Independent Researcher

dacquarone@bignottiedacquarone.it

https://orcid.org/0000-0002-3837-8178

\begin{abstract}
Lo scritto analizza il ruolo assegnato dal legislatore all'ente nella lotta alla corruzione, dedicando particolare attenzione alla previsione contenuta nel comma 5-bis dell'art. 25 (concussione, induzione indebita a dare o promettere utilità e corruzione) d. Igs. 8 giugno 2001, n. 231. Di questa disposizione si segnalano le criticità applicative di natura pratica e le loro principali cause, tra cui la crescente tendenza a sovraccaricare l'ente di oneri cooperativi non sufficientemente ricompensati, che, anche secondo una lettura costituzionalmente orientata, dovrebbero, quanto meno, essere egualmente ripartiti con le autorità pubbliche. La soluzione prospettata è quella raccomandata dal vertice G20 di Los Cabos (Messico) del 17 giugno 2012: elaborare un framework di riferimento
\end{abstract}

1 Avvocato del Foro di Verona e Cultore della materia in Diritto processuale penale presso il Dipartimento di Scienze Giuridiche dell'Università degli Studi di Verona.

2 Avvocato del Foro di Verona, Solicitor UK e Corresponsabile dell'Osservatorio d. lgs. 231/2001 dell'Unione delle Camere Penali Italiane. 
chiaro e concreto che disciplini esattamente i presupposti, i termini e i vantaggi della cooperazione dell'ente nelle indagini dell'autorità giudiziaria, sì da garantire un equilibrato e accettabile bilanciamento tra efficienza preventivo-repressiva ed esigenze di libertà per l'ente e all'interno dell'ente.

PARole-chiave: Lotta alla corruzione; cooperazione dell'ente; diritti e doveri dell'ente; indagini dell'autorità giudiziaria; sistemi premiali.

ABSTRACT: The paper analyzes the role assigned by the legislator to the entity in the fight against corruption, paying attention to the provision contained in paragraph 5-bis of art. 25 (bribery, undue induction to give or promise benefits and corruption) d. Igs. June 8, 2001, n. 231. The essay highlights the practical application criticalities of this provision and their main causes, among which the growing tendency to overload the institution with insufficiently rewarded cooperative burdens, which, even according to a constitutionally oriented reading, should, at the very least, to be equally shared with public authorities. The solution proposed is the one recommended by the B20 summit in Los Cabos (Mexico) on 17 June 2012: developing a clear and concrete reference framework that exactly disciplines conditions, terms and advantages of the institution's cooperation in the judicial authority investigations, so as to guarantee an equilibrated and acceptable balance between preventive-repressive efficiency and freedom needs for the institution and within the institution.

KEYwORDS: Fight against corruption; cooperation of the entity; rights and duties of the entity; judicial authority investigations; reward systems.

SOMmario: 1. Introduzione: il comma 5-bis dell'art. 25 d. Igs. 8 giugno 2001, n. 231. Alcune criticità in superficie. - 2. La ratio della circostanza attenuante nelle sue molteplici profondità. 3. L'oggetto del presente lavoro. - 4. Gli elementi dissuasori interni all'ente. - 5. Gli elementi dissuasori esterni all'ente. L'atteggiamento (presumibilmente) ostile della magistratura. - 6. La causa maggiore della crisi del sistema cooperativo e, più in generale, dell'impianto normativo della responsabilità dell'ente. - 7. Quale antidoto all'inefficacia del sistema cooperativo abbozzato nel comma 5-bis dell'art. 25 d. Igs. 231/2001? - 8. Conclusioni provvisorie. Bibliografia. 


\section{INTRODUZIONE: IL COMMA 5-BIS DELL'ART. 25 D. LGS. 8 GIUGNO 2001, N. 231. AlCUNE CRITICITÀ IN SUPERFICIE.}

Nell'insieme di novità che si registra a opera della 1.9 gennaio 2019 , n. 3, che ha introdotto modifiche all'art. 25 d. lgs. 8 giugno 2001, n. 231, quella di maggiore respiro, sotto il profilo della politica sanzionatoria a carico dell'ente, è forse quella contenuta nel nuovo comma 5-bis inserito nel menzionato art. 25. L'interpolazione prevede che, «se prima della sentenza di primo grado l'ente si è efficacemente adoperato per evitare che l'attività delittuosa sia portata a conseguenze ulteriori, per assicurare le prove dei reati e per l'individuazione dei responsabili ovvero per il sequestro delle somme $\mathrm{o}$ altre utilità trasferite e ha eliminato le carenze organizzative che hanno determinato il reato mediante l'adozione e l'attuazione di modelli organizzativi idonei a prevenire reati della specie di quello verificatosi, le sanzioni interdittive hanno la durata stabilita dall'articolo 13, comma $2 »$, vale a dire fino a 2 anni. Il comma 5-bis dell'art. 25 d. lgs. 231/2001 introduce, così, una nuova circostanza attenuante a effetto speciale.

Il funzionamento del meccanismo sembra intuitivo: sanzioni gravi o gravissime per l'ente non virtuoso né cooperativo; rientro delle sanzioni nella cornice edittale ordinaria per l'ente che, oltre a innovare la propria organizzazione, si adoperi prima della condanna cooperando efficacemente con l'autorità giudiziaria ${ }^{3}$. Il senso ultimo di questo schema può essere così compendiato: lo Stato stringe una partnership con il mondo delle imprese nella «cogestione dei rischi legati alla criminalità d'impresa» al fine di «giungere alla più efficace prevenzione dell'illecito economico con i minori oneri possibili, rispetto allo scopo, a carico

3 Sottolinea SERENI, Andrea. Colpa e cooperazione investigativa nella responsabilità dell'ente da reato. Un confronto tra ordinamenti nella prospettiva della globalizzazione, Rivista trimestrale di diritto penale dell'economia, v. 3-4, p. 635-636, 2018 che «lo schema è piuttosto semplice: garantire alle imprese vantaggi, in termini di trattamento penale, in cambio di un impegno fattivo nel debellamento della criminalità economica e nella dimostrazione di una totale lealtà verso le autorità inquirenti nella ricerca dei soggetti responsabili dei reati commessi all'interno delle strutture aziendali. Una giustizia negoziata, quindi, in funzione dell'ottenimento di risultati efficaci nella prevenzione/repressione della criminalità d'impresa; l'ente ha il vantaggio [...] di un trattamento sanzionatorio più favorevole». 
delle organizzazioni private» ${ }^{4}$. Muovendo dal presupposto che le attività pubbliche di prevenzione brancolano di fronte alla complessità dei mercati globali e, in particolare, dei sistemi di funzionamento aziendali, la strategia eletta è quella di alterare, per così dire, la natura dell'ente, trasformandolo in un "tutore dell'ordine" e in un "guardiano della legalità"

A margine del presente lavoro, alcune prime notazioni critiche sull'impatto pratico del sistema di premialità sul piano della responsabilità dell'ente ${ }^{6}$. La scelta legislativa sembra trascurare la natura fondamentalmente accessoria di questa responsabilità, che frequentemente presuppone l'esistenza del reato in capo a una persona fisica già determinata. Infatti, una delle condizioni di accesso alla circostanza attenuante in argomento è rappresentata dall'essersi l'ente efficacemente adoperato per l'individuazione dei responsabili. Circostanza, questa, che nella prassi precede, fuori del caso, comunque residuale, di cui all'art. 8 comma 1 lett. $a$ d. lgs. 231/2001, l'annotazione dell'illecito amministrativo ex art. 55 d. lgs. 231/2001. All'inverso, se i responsabili del reato-presupposto non sono ancora stati individuati ed è l'ente a rivelarne l'identità, dalla denuncia può scaturire anche il procedimento a carico dell'ente. L'effetto è che se il procedimento penale è già instaurato, sembrano ritagliarsi spazi assai modesti per un'utile cooperazione processuale, risultando verosimilmente già individuati l'autore materiale del reato-presupposto e le somme o le altre utilità trasferite. Viceversa, se l'autorità giudiziaria

4 CENTONZE, Francesco. Responsabilità da reato degli enti e agency problems. I limiti del d.lgs.vo n. 231 del 2001 e le prospettive di riforma. Rivista italiana di diritto e procedura penale, v. 3, p. 986, 2017.

5 JENNIFER, Arlen. L'applicazione della legge penal-societaria negli Stati Uniti: l'uso delle transazioni per trasformare imprese potenzialmente criminali in tutori dell'ordine. Rivista trimestrale di diritto penale dell'economia, v. 1-2, p. $1,2018$.

6 FIORELLA, Antonio. Logica, criticità e riforme del sistema punitivo per l'illecito dell'ente da reato. Rivista italiana di diritto e procedura penale, v. 1, p. 530, 2019; MASULLO, Maria Novella. L'emersione del patto corruttivo: il nuovo fronte degli strumenti premiali e investigativi. Rivista italiana di diritto e procedura penale, v. 3, p. 1276-1278, 2019; PIERGALLINI, Carlo. Premialità e non punibilità nel sistema della responsabilità da reato degli enti. Diritto penale e processo, v. 4, p. 530, 2019; SANTORIELLO, Ciro. Anche nei confronti degli enti collettivi il decreto anticorruzione mostra inutili e incoerenti “furori sanzionatori”. Diritto penale e processo, v. 6, p. 756, 2019. 
non ha ancora avuto notizia del reato-presupposto, raramente all'ente converrà cooperare denunciando il fatto di reato e i connessi responsabili, potendo giovarsi della sola riduzione dei limiti edittali delle sanzioni interdittive e non già dell'esonero da responsabilità.

Insomma, non precisando la 1. 3/2019 i limiti di esigibilità dell'iniziativa di cooperazione che l'ente può assumere, ci si interroga, in prima battuta, se quest'ultimo possa beneficiare dello "sconto" di pena anche nel caso in cui i responsabili siano già stati individuati e le prove del reato-presupposto siano già state assicurate. Del pari, ci si domanda se l'ente possa egualmente usufruire della circostanza attenuante in questione nel caso in cui, nonostante il suo impegno spontaneo, la ricerca dei responsabili e delle prove si riveli inconcludente. Sembra di no in ragione della presenza dell'avverbio «efficacemente».

Peraltro, il rischio di ineffettività della circostanza attenuante in esame sembra potenziato dal fatto che il d. lgs. 231/2001 è già disseminato di numerose disposizioni che esaltano, in termini di mitigazione della responsabilità e di sospensione delle misure cautelari eventualmente applicate, le iniziative riparatorie e cooperative assunte dall'ente nelle diverse fasi del procedimento. Disposizioni che già garantiscono all'ente, ma con rischi questa volta minori, il conseguimento delle medesime finalità premiali sottese alla circostanza attenuante di cui al comma 5-bis dell'art. 25 d. lgs. 231/2001.

Al di là delle criticità rilevate a una prima lettura della norma in argomento, rimane che la legge, attestando una reazione dell'ordinamento particolarmente ferma ai fenomeni corruttivi, condiziona l'ammissione dell'ente alla sanzione ridotta all'efficace cooperazione, gravando lo stesso di ogni accertamento utile all'individuazione dell'autore materiale del reato-presupposto e all'assicurazione delle prove, vale a dire di attività essenzialmente vicarie rispetto a quelle del pubblico ministero e degli organi di polizia. Insomma, le unità lavorative e i relativi presidi di vigilanza sembrano venire adibiti al «ruolo di "collaboratori sul territorio" della polizia giudiziaria e del pubblico ministero» ${ }^{7}$.

7 SERENI, Andrea. Colpa e cooperazione investigativa nella responsabilità dell'ente da reato. Un confronto tra ordinamenti nella prospettiva della globalizzazione. Rivista trimestrale di diritto penale dell'economia, v. 3-4, p. 641, 2018. 


\section{La RATIO DELLA CiRcostanza ATtENUANTE NELLE SUE MOLTEPLICI PROFONDITÀ.}

Il comma 5-bis dell'art. 25 d. lgs. 231/2001 si inserisce in un filone tematico che vede il legislatore degli ultimi tempi rinunciare gradualmente agli ultimi indugi di matrice culturale e porre l'opzione premiale alla base delle scelte di politica criminale. La preferenza trova diffuse adesioni, probabilmente favorite dal mutato clima di ampio consenso per le manifestazioni espressive di un approccio di non connivenza nel reato ${ }^{8}$. Peraltro, la strategia del premio per la cooperazione processuale ${ }^{9}$, atta a valorizzare il ruolo sostanziale della denuncia, è da tempo efficacemente collaudata in altri settori: terrorismo, criminalità organizzata, sequestro di persona a scopo estorsivo e traffico di stupefacenti. Sicché l'ampliamento al catalogo dei reati-presupposto contro la pubblica amministrazione sembra convalidare il progressivo - censurabile, ad avviso di chi scrive «allineamento normativo della criminalità white collar alla criminalità nera, ossia della criminalità politico-economica al crimine organizzato» ${ }^{10}$.

8 Ex plurimis, PELLISSERO, Marco. La nuova disciplina della corruzione tra repressione e prevenzione. In: MATTARELLA, Bernardo Giorgio-PELLISSERO, Marco (a cura di). La legge anticorruzione. Torino: Giappichelli, 2013. p. 351.

9 AMARELLI, Giuseppe. L'attenuante della dissociazione attuosa (art. 8 d.l. n. 152/1991). In: MAIELLO, Vincenzo (a cura di). La legislazione penale in materia di criminalità organizzata, misure di prevenzione e armi. Torino: Giappichelli, 2015. p. 163-164; RUGA RIVA, Carlo. Il premio per la collaborazione processuale. Milano: Giuggrè, 2002.

10 MANES, Vittorio. L'estensione dell'art. 4-bis ord. pen. ai delitti contro la P.A.: profili di illegittimità costituzionale. Rivista trimestrale di diritto penale contemporaneo, v. 2, p. 107, 2019. Corre l'obbligo di rilevare che autorevole dottrina da tempo ritiene, per converso, che i reati "economici" rappresentino, in realtà, un terreno su cui la criminalità dei "colletti bianchi" e la criminalità organizzata tradizionale possono convergere e, a volte, incontrarsi: v., per tutti, a livello internazionale, SUTHERLAND, Edwin Hardin. White Collar Criminality. American Sociological Review, v. 5, n. 1, p. 1-12, 1940; SUTHERLAND, Edwin Hardin. White Collar Crime: The Uncut Version. New Heaven: Yale University Press, 1983; per una panoramica delle teorie criminologiche, v. RACITI, Annamaria. Il criminale economico nella ricerca criminologica: dall'opera di Sutherland alle più recenti formulazioni teoretiche. Rivista trimestrale di diritto penale dell'economia, v. 3, p. 677-699, 2005; infine, v., nella letteratura italiana, FORTI, Gabrio. L'immane concretezza: metamorfosi del crimine e controllo penale. Milano: Raffaello Cortina Editore, 2000; 
Ciò atteso, la ratio della circostanza attenuante in questione sembra potersi rintracciare, in un primo livello, nella necessità di incrementare le denunce di fatti corruttivi e, quindi, di accrescere il numero delle relative condanne, ricompensando l'ente delatore e cooperativo per avere interrotto il vincolo collusivo caratteristico delle fattispecie corruttive, consentendo, per questa via, la perseguibilità anche della persona fisica. Perciò, la novità legislativa si pone solo apparentemente in controtendenza rispetto alla politica penale degli inasprimenti sanzionatori caldeggiata dal legislatore più recente. All'opposto, essa sembra aderire alla medesima logica di consolidamento dell'intervento punitivo, immersa, tuttavia, in una dimensione di utilità collettiva che sembra volere salvaguardare, fino a dove possibile, «l'esigenza di prevenzione generale, in quanto idonea a ribadire l'efficacia del precetto e convalidarne "esemplarmente" il significato» ${ }^{11}$.

PALIERO, Carlo Enrico. Criminalità economica e criminalità organizzata: due paradigmi a confronto. In: BARILLARO, Michele (a cura di). Criminalità organizzata e sfruttamento delle risorse territoriali. Milano: Giuffrè, 2004. p. 141 ss.; VANNUCCI, Alberto. La corruzione nel sistema politico italiano a dieci anni da "mani pulite". In: FORTI, Gabrio (a cura di). Il prezzo della tangente: la corruzione come sistema a dieci anni da "mani pulite". Milano: Vita e Pensiero, 2003. p. 1-68.

11 Per un approfondimento, più in generale, sul tema della "dissociazione" dal patto corruttivo, che in questa sede, per ragioni di economia del presente lavoro, appena si accenna, v., per tutti, DI MARTINO, Alberto. La sequenza infranta: profili della dissociazione tra reato e pena. Milano: Giuffrè, 1998; PADOVANI, Tullio. Il traffico delle indulgenze. "Premio" e "corrispettivo" nella dinamica della punibilità. Rivista italiana di diritto e procedura penale, v. 2, p. 398-436, 1986. Certamente negativo, seppure con diversità di accenti, il giudizio dei giuristi dell'Illuminismo e dei Maestri della penalistica italiana, che tradizionalmente respingono l'idea che l'ordinamento possa, in qualsiasi modo, offrire "ponti d'oro" a chi si ravveda e si penta del fatto commesso, come controspinta psicologica. Sul punto, v. FILANGIERI, Gaetano. La Scienza della Legislazione. Napoli: Grimaldi \& C., 2003, secondo il quale «molto meno ammetter si dovrebbe come un ragionevol motivo d'impunità il perdono, che si suol promettere ad un complice per la scoverta degli altri. Quando la sanità delle leggi non fosse incompatibile con un rimedio, che ha il più vile tradimento per mezzo; quando non fosse un indizio di debolezza d'impotenza, il vedere che la legge implora l'ajuto di chi l'offende; quando l'esperienza non ci avesse mostrato, che in questi casi il più malvagio è ordinariamente quegli, che scampa il rigore della pena; la sola ragione bastar dovrebbe per distogliere il legislatore dal ricorrere a questo rimedio, il quale 
Scendendo in profondità, la circostanza attenuante in esame può, in un secondo livello, produrre un effetto di deterrenza determinato dall'«introduzione di un fattore di insicurezza» ${ }^{12}$ all'interno del patto corruttivo, reso incerto proprio dalla possibilità per l'ente che denunci il fatto di garantirsi uno "sconto" di pena. Sicché sembra confermarsi una premialità giustificata dall'opportunità - o dalla necessità - di rimuovere gli impedimenti alla repressione del reato determinati dall'abitualmente complicata emersione del fatto corruttivo ${ }^{13}$.

non solo è inefficace a produrre l'effetto che si desidera, ma può divenire la causa dell'effetto opposto. La speranza o la sicurezza, dell'impunità concessa alla delazione del complice, invece di distogliere, renderà più ardito il malvagio avveduto ad intraprendere il delitto, cha ha bisogno del concorso di più uomini. Prima di sedurre i suoi compagni all'intrapresa del delitto, egli ha già concepito il pravo disegno d'immolarli alla sua sicurezza, quando vedesse prossima la scoverta dei rei. Ciascheduno dei suoi compagni, prima di aderire, formerà l'istesso disegno. La speranza dell'impunità allignerà ugualmente in tutti questi perfidi cuori, e gli renderà più arditi all'intrapresa. Ciascheduno vedrà nella delazione il suo sicuro asilo, e con questa lusinga il terrore della pena sarà ugualmente indebolito in ciascheduno dei complici dalla comune speranza dell'impunità; il delitto sarà incoraggiato dal mezzo istesso, che la legge adopera per punirlo; ed il legislatore deluso nelle sue speranze vedrà con rimorso i funesti effetti di un rimedio, che, ancorché utile, dovrebbe essere abbandonato come contrario alla veneranda dignità delle leggi»; CARRERA, Francesco. Programma del corso di diritto criminale: parte generale. Lucca: Il Mulino, 1871, secondo il quale «da un lato ripugna che la legge dica in precedenza che quando un accusato confesserà a danno proprio o dei complici, avrà uno sgravio di pena. Questo dettato apparisce una transazione col delitto. [...] Dall'altro lato è un fatto, che se l'utilità politica può tenersi come giusta ragione per ammolire a riguardo di lei alcun poco il rigore della pena, questa utilità derivante dalla confessione di un accusato, non può raggiungersi né congruamente valutarsi ove la cognizione della spontaneità o della importanza della confessione giudiciale non differisca ai magistrati in faccia ai quali si agita il processo». Infine, per una riflessione generale sul rapporto tra reato e pena, v. HASSEMER, Winfried. Perché punire è necessario. Bologna: Il Mulino, 2012.

12 PULITANÒ, Domenico. Le cause di non punibilità dell'autore della corruzione e dell'infiltrato e la riforma dell'art. 4-bis. Diritto penale e processo, v. 5, p. 601, 2019.

13 DI MARTINO, Alberto. La sequenza infranta: i profili della dissociazione tra reato e pena. Milano: Giuffrè, 1998; MAIELLO, Vincenzo. Fuga dalla sanzione e postmodernità penalistica. In: MOCCIA, Sergio (a cura di). La giustizia contrattata: dalla bottega al mercato globale. Napoli: Edizioni Scientifiche Italiane, 1998. p. 115 ss. 
Al termine della discesa, il peso che il comma 5-bis dell'art. $25 \mathrm{~d}$. lgs. 231/2001 contemporaneamente assegna all'attività riparatoria - «se prima della sentenza di primo grado l'ente si è efficacemente adoperato per evitare che l'attività delittuosa sia portata a conseguenze ulteriori [...] e ha eliminato le carenze organizzative che hanno determinato il reato mediante l'adozione e l'attuazione di modelli organizzativi idonei a prevenire reati della specie di quello verificatosi» - sembra mirare a incoraggiare l'ente a rendere concretamente «operativo un modello organizzativo idoneo a prevenire reati della specie di quello verificatosi» (art. 12 comma 2 lett. $b$ d. lgs. 231/2001) e a un pieno "ritorno alla legalità”. Dunque, «il virtuoso comportamento dell'ente» sembra essere «il reale obiettivo delle sanzioni [attenuate]: vero "perno" attorno a cui ruota l'intera, relativa disciplina sanzionatoria, rappresentando il fattore di "equilibrio" del sistema di recupero alla normalità dell'attività collettiva, così permettendo di escludere conseguenze punitive esorbitanti» ${ }^{14}$.

\section{L'oggetTo dEL PRESENTE LAVORO.}

Alla luce di quanto sopra, si impone, da un punto di vista sia dogmatico sia pratico, la necessità di una seria verifica delle reali cause del fallimento, agevolmente prevedibile, del sistema cooperativo tracciato nel comma 5-bis dell'art. 25 d. lgs. 231/2001. Al contempo, si pone il quesito se e in quale misura l'attuale sistema possa, con le opportune revisioni, incentivare, in modo equilibrato ma efficace ed efficiente, l'emersione dei patti corruttivi connessi alle attività dell'organizzazione pluripersonale nel pieno rispetto, oltreché dei doveri, dei diritti di tutte le parti in causa.

\section{GLI ELEMENTI DISSUASORI INTERNI ALL'ENTE.}

In primo luogo, difficilmente l'ente troverà conveniente elaborare, ad esempio all'interno del codice etico o della parte generale del modello

${ }^{14}$ FIORELLA, Antonio. Logica, criticità e riforme del sistema punitivo per l'illecito dell'ente da reato. Rivista italiana di diritto e procedura penale, v. 1 , p. 521, 2019. 
organizzativo, un percorso predefinito di cooperazione con l'autorità giudiziaria azionabile nel caso in cui l'ente stesso scopra, ad esempio in seguito a una segnalazione interna, la commissione di uno dei reatipresupposto di cui all'art. 25 d. lgs. 231/2001. Troppe e troppo gravi le controindicazioni di ordine pratico determinate dal rischio di un impiego distorto di un simile sistema, che, specie all'interno di strutture aziendali di medio-grandi dimensioni, potrebbe sensibilmente peggiorare la qualità di lavoro delle persone, assumendo, così, contorni assai poco rispondenti al volto liberale e democratico del diritto penale costituzionale ${ }^{15}$.

In particolare, e a titolo meramente esemplificativo, questo sistema potrebbe favorire la creazione di "capri espiatori". Infatti, non è, francamente, da escludere che «l'organizzazione possa "sacrificare" il singolo per "salvare" l'ente, canalizzando verso l'individuo responsabilità anche della persona giuridica» ${ }^{16} \mathrm{e}$, per questa via, distogliendo l'attenzione dall'eventuale inefficienza preventiva della politica aziendale interna. Per altro verso, questo sistema potrebbe indurre a una sorta di "caccia all'uomo". Infatti, è possibile che, perfino nei casi più incerti sotto il profilo della configurazione stessa della responsabilità dell'ente, la prospettiva di premi significativi, ancorché non risolutivi, per la cooperazione nelle indagini spinga l'ente stesso a investigare comunque sull'autore del reatopresupposto invece di coltivare la propria difesa, accettando, sin da subito, l'ancorché dubbia ipotesi accusatoria formulata dal pubblico ministero al fine di guadagnare un esito del procedimento, se non più conveniente, senz'altro più celere e certo. A ciò si unisca che questo sistema non proteggerebbe l'ente dall'onere del travaglio reputazionale, che, comunque, accompagnerebbe l'emersione del reato-presupposto. Ciocché, all'inverso, rappresenterebbe per l'ente un forte e rilevante incentivo a un'inerzia deliberata nell'attivazione della cooperazione con l'autorità giudiziaria.

15 MOCCIA, Sergio. La "promessa non mantenuta": ruolo e prospettive del principio di determinatezza/tassatività nel sistema penale italiano. Napoli: Edizioni Scientifiche Italiane, 2001; PATRONO, Paolo. Diritto penale dell'impresa e interessi umani fondamentali. Padova: Cedam, 1993.

16 CENTONZE, Francesco. Responsabilità da reato degli enti e agency problems. I limiti del d.lgs.vo n. 231 del 2001 e le prospettive di riforma. Rivista italiana di diritto e procedura penale, v. 3, p. 686-687, 2017. 
Circostanze, queste, progressivamente destinate ad assottigliare la voce dei benefici e, per converso, a impinguare quella dei costi connessi alla cooperazione con l'autorità giudiziaria, così riducendo considerevolmente le chance di complessivo successo dell'istituto.

\section{GLI ELEMENTI DISSUASORI ESTERNI ALL'ENTE. L'ATTEGgIAMENTO (PRESUMIBILMENTE) OSTILE DELLA MAGISTRATURA.}

In secondo luogo, anche nel caso in cui l'ente si determinasse a favore della cooperazione con l'autorità giudiziaria, non può dirsi scontato che l'iniziativa collaborativa conseguentemente assunta dall'ente stesso superi fattibilmente il vaglio di efficacia, sollecitato dal comma 5-bis dell'art. 25 d. lgs. 231/2001, atteso l'atteggiamento tutt'ora di sostanziale chiusura e sospetto della magistratura nei confronti degli enti incolpati, spesso avvertiti come soggetti che hanno nel proprio DNA un'inarrestabile tendenza a corrompere.

Il pensiero inevitabilmente corre alla ben nota visione della Procura della Repubblica di Milano, ufficio tra i più attivi nell'applicazione del d. lgs. 231/2001, espressa - e, forse, determinata - da Francesco Greco proprio nell'ambito della lotta alla corruzione. Infatti, Greco sostiene che, attesi il «fallimento dei controlli interni», «che non hanno [...] dato prova di esistenza», e l'«inerzia o inefficienza dimostrata dai controlli preventivi», «non deve sorprendere la constatazione che, negli ultimi venti anni, l'unico efficace strumento di controllo sulle società e sui mercati sia stato quello penale». Peraltro, la posizione di Greco è - come lui stesso riconosce con una certa franchezza - quella caratteristica del pubblico ministero, che, «per una sorta di deformazione professionale, finisce per ravvisare illeciti in ogni manifestazione sociale ${ }^{17}$. Per glissare sul fatto che «rarissime sono [...] le pronunzie di merito che hanno prosciolto l'ente in ragione dell'adozione dei [...] modelli, alcune delle quali nemmeno hanno superato il vaglio di legittimità», stante il perdurare dell'«inaccettabile

17 GRECO, Francesco. Perché "fallisce" il sistema dei controlli? In: BRESCIA, Franco-TORCHIA, Luisa-ZOPPINI, Andrea (a cura di), Metamorfosi del diritto delle società?: seminario per gli ottant'anni di Guido Rossi. Napoli: Editoriale Scientifica, 2012, p. 127-128 e 131. 
equazione per cui la realizzazione del reato presupposto sarebbe di per sé la prova dell'inadeguatezza del programma di compliance» ${ }^{18}$.

Se l'ottica con cui l'autorità giudiziaria guarda alla lotta alla corruzione è non neutrale ma suggestionata da sbrigativi preconcetti, in quale accoglienza potrà mai confidare l'ente cooperativo? Infatti, un atteggiamento diffidente potrebbe, nella migliore delle ipotesi, indurre l'autorità giudiziaria a non vedere con fiducia e, quindi, a non considerare la cooperazione dell'ente nelle indagini o, all'opposto, e in modo peggiore, a considerare la mancata cooperazione alla stregua di un «indizio di opacità e ambiguità di comportamento della persona giuridica». Peraltro, il rischio sarebbe, in quest'ultimo caso, che «lo stesso esercizio del diritto di difesa, inteso anzitutto come diritto al silenzio e alla non collaborazione, venga valutato dal giudice come colpa per omessa reazione all'illecito», vale a dire come «sintomo a posteriori della inefficace attuazione del modello preventivo eventualmente adottato». Per questa via, «potrebbe offuscarsi la stessa linea di demarcazione tra rischio consentito, legato all'esercizio delle proprie prerogative difensive, e rischio non consentito sanzionabile» ${ }^{19}$.

Occorre, naturalmente, distinguere caso per caso, ma «un atteggiamento a priori negativo nei confronti delle misure prese dalle imprese private, certo non agevola una efficace lotta alla corruzione $»^{20}$.

\section{LA CAUSA MAGGIORE DELLA CRISI DEL SISTEMA COOPERATIVO E, PIÙ IN GENERALE, DELL'IMPIANTO NORMATIVO DELLA RESPONSABILITÀ DELL'ENTE.}

In realtà, quelli segnalati nei paragrafi 4 e 5 che precedono descrivono soltanto alcuni indicatori di un malessere più diffuso patito

18 PISTORELLI, Luca. La responsabilità da reato degli enti: un bilancio applicativo. Rivista trimestrale di diritto penale dell'economia, v. 3-4, p. 616-617, 2017.

19 SERENI, Andrea. Colpa e cooperazione investigativa nella responsabilità dell'ente da reato. Un confronto tra ordinamenti nella prospettiva della globalizzazione. Rivista trimestrale di diritto penale dell'economia, v. 3-4, p. 640, 2018.

20 BONELLI, Franco. "Collaborazione" tra autorità pubbliche e società private per un'efficace lotta alla corruzione. Diritto del commercio internazionale, v. 3, p. $678,2012$. 
dal sistema cooperativo tracciato nel comma 5-bis dell'art. 25 d. lgs. 231/2001 e, più in generale e ancora prima, dalla disciplina tutta della responsabilità dell'ente.

Nella prospettiva di prevenzione, in particolare dei fenomeni corruttivi, all'ente è richiesto di esercitare una sorveglianza sempre più minuziosa, continua e ingombrante, mediante «un crescendo di protocolli preventivi da osservare sempre più rigidi $[. .$.$] in corrispondenza$ dei molteplici scopi cautelari fissati dalla legge ${ }^{21}$, ivi compresa l'anticorruzione. In questo senso, è, al contempo, emblematico e allarmante il dato cronologico-oggettivo: dal 2001, anno di entrata in vigore del d. lgs. 231/2001, al 2012, anno di entrata in vigore della 1.6 novembre 2012, n. 190, recante disposizioni per la prevenzione e la repressione della corruzione e dell'illegalità nella pubblica amministrazione, la gestione, in termini di prevenzione, dei delitti di matrice corruttiva è stata posta a carico esclusivo dell'ente privato.

Sennonché la proliferazione e l'intensificazione di cautele e la ramificazione della vigilanza rischiano, mediante congegni più insidiosi che in passato (incentivazione delle segnalazioni e, oggi, sollecitazione alla cooperazione con l'autorità giudiziaria), di stringere ulteriormente le già strette corde della "camicia di forza" disciplinare applicata all'ente da un legislatore ancora innamorato dei tratti della «società punitiva» ${ }^{22}$ tipici degli anni Settanta del secolo scorso.

A questo punto, c'è, allora, da interrogarsi se la venuta della «modernità liquida ${ }^{23}$ abbia effettivamente comportato per l'ente il

21 SERENI, Andrea. Colpa e cooperazione investigativa nella responsabilità dell'ente da reato. Un confronto tra ordinamenti nella prospettiva della globalizzazione. Rivista trimestrale di diritto penale dell'economia, v. 3-4, p. 626, 2018.

22 FOUCAULT, Paul-Michel. La società punitiva: corso al Collège de France (1972-1973). Milano: Feltrinelli, 2016; FOUCAULT, Paul-Michel. Sorvegliare e punire: nascita della prigione. Torino: Einaudi, 1993; nello specifico ambito della responsabilità degli enti, v. SERENI, Andrea. L'ente guardiano: l'autorganizzazione del controllo penale. Torino: Giappichelli, 2016.

23 BAUMAN, Zygmunt. Modernità liquida. Roma-Bari: Editori Laterza, 2011 osserva che «gli abitanti del Panopticon erano incatenati al loro posto e impossibilitati a muoversi, [...] legati ai loro letti, celle o banchi di lavoro. Non potevano muoversi perché erano sotto stretta osservazione [...] La fine del Panopticon preconizza la fine dell'epoca del reciproco coinvolgimento: tra 
definitivo superamento del "modello panottico", vale a dire del modello fondato su un rapporto di stretta osservazione tra controllore e controllato.

A riflettere sul progresso del diritto penale odierno sembra, nondimeno, che l'evoluzione su larga scala verso una fase "post-panottica" possa dirsi tutt'altro che confermata per l'ente. Infatti, l'approccio legislativo e giurisprudenziale attesta, in questo settore sociale strategico, che il "modello panottico" non è stato affatto rimpiazzato con paradigmi nuovi, ma va, piuttosto, progredendo in qualcosa di più articolato e, forse, di più penetrante del primigenio Panopticon. Per la responsabilità dell'ente, si può parlare, all'inverso, di implementazione di un "modello neo-panottico", il cui paradigma punitivo mira a prospettare un criterio di controllo interno "totale" e "circolare". Infatti, rispetto al tradizionale "modello panottico", dall'ente si esige un controllo non più solo, o primariamente, organizzato in senso gerarchico "top-down", ma progettato anche in modo tale che le informazioni siano convogliate "bottom-up", sì che il controllato possa, a sua volta, vigilare sul controllore (c.d. "whistleblowing” di cui all'art. 6 commi 2-bis, 2-ter e 2-quater d. lgs. 231/2001), e si propaghino in senso orizzontale all'esterno, in modo appunto da includere, per lo meno occasionalmente nelle ipotesi più gravi, anche l'autorità giudiziaria entro la circonferenza del microcosmo aziendale. In questo senso, è emblematico lo sforzo corale richiesto all'ente dal comma 5-bis dell'art. 25 d. lgs. 231/2001 nel senso di una denuncia tempestiva degli illeciti di matrice corruttiva eventualmente scoperti ${ }^{24}$.

Per queste ragioni, sia pure ricordate per sintesi, sembra che il rischio di una deriva "neo-panottica" si sia già concretato a causa della corrente ideologia che esalta, in particolare rispetto al fenomeno corruttivo, l'efficienza tecnico-scientifica della prevenzione e l'efficacia repressiva della reazione. Per fare solo un esempio, si pensi alla prospettata ipotesi di immaginare «una

controllori e controllati, capitale e lavoro, leader e seguaci. La principale tecnica di potere diventa ora la fuga, l'evasione, il distacco, il netto rifiuto di qualsiasi confinamento territoriale».

24 Per un raffronto con il diritto statunitense, v. MAZZACUVA, Federico. La diversione per gli enti collettivi nell'esperienza anglo-americana. Alcuni spunti de iure condendo. Rivista trimestrale di diritto penale contemporaneo, v. 2, p. 81, 2016; RUGGIERO, Rosa Anna. Non prosecution agreement e criminalità d'impresa negli U.S.A.: il paradosso del liberismo economico. Diritto penale contemporaneo, p. 8, 12 ottobre 2015. 
strategia "collaborativa" che includa, nei programmi di compliance, procedure di selezione dei manager e del personale in base alla loro "propensione al reato", da valutare alla luce di "test [psicologici] ormai corroborati", in grado di svelare i corporate psycopaths, e di mettere a nudo "furbizia" e "capacità di manipolazione" nel "tenere il comportamento che fa una migliore impressione sul posto di lavoro e durante i colloqui professionali” $»^{25}$.

A ciò si coniughi la tendenza in atto a modellare l'organizzazione aziendale sull'archetipo del bad man, muovendo dall'assunto che nella personalità di ciascuno alberga un "lato oscuro" in grado di coltivare propositi criminosi ${ }^{26}$. La prevenzione e la reazione al fenomeno corruttivo vengono, allora, articolate in funzione della "normalità della devianza" invece che della normalità del comportamento socialmente corretto ${ }^{27}$. Per questa via, il modello organizzativo e l'attività di vigilanza guadagnano un surplus di coartazione, anche simbolica, dando origine a una corsa improduttiva alla prevenzione e alla trasparenza "totali".

\section{Quale ANTIDOTO ALL'INEFFICACIA DEL SISTEMA COOPERATIVO ABBozZATO NEL COMMA 5-BIS DELL'ART. 25 D. LGS. 231/2001?}

Chi scrive si trova, suo malgrado, d'accordo con la previsione di chi ritiene che «questa "chiamata alle armi" contro la criminalità

25 BERTOLINO, Marta. Dall'organizzazione all'individuo: crimine economico e personalità, una relazione da scoprire. Criminalia, p. 31-32, 2014, commentata anche in SERENI, Andrea. Colpa e cooperazione investigativa nella responsabilità dell'ente da reato. Un confronto tra ordinamenti nella prospettiva della globalizzazione. Rivista trimestrale di diritto penale dell'economia, v. 3-4, p. 626-627, 2018.

26 GARAPON, Antoine. La responsabilità delle persone giuridiche e le nuove "regole del gioco" mondiale. I paradigmatici casi BNP-Paribas e Alstom. In: CENTONZE, Francesco-MANTOVANI, Massimo (a cura di). La responsabilità "penale" degli enti: dieci proposte di riforma. Bologna: Il Mulino, 2016. p. 42, secondo cui «bisogna purtroppo fare i conti con le naturali tendenze dell'uomo comune: il diritto deve sempre presupporre di avere a che fare col bad man, e non c'è linea guida, codice di condotta o "carta etica" che possa sostituirsi all'applicazione della legge penale».

27 BIANCHETTI, Raffaele. La paura del crimine: un'indagine criminologica in tema di mass media e politica criminale ai tempi dell'insicurezza. Milano: Giuffrè, p. 217, 2018. 
affaristica sembra destinata a divenire il nocciolo duro del rimprovero penale e dell'irrogazione, in caso d'inadempienza, della sanzione a carico dell'ente collettivo». Infatti, «la colpa di questa "comunità", di questo aggregato di persone fisiche, consiste, a questo punto, nel restare inattivi o nel non attivarsi in modo adeguato contro i rischi interni di condotte criminali $»^{28}$, per prima cosa autodenunciandosi.

Ciò atteso, il rimedio alle criticità riassunte nei paragrafi che precedono è semplice: occorre mutare prospettiva. La lotta alla corruzione richiede uno sforzo e un impegno comuni, vale a dire non solo dell'ente privato, ma anche della parte pubblica, ognuno per il proprio ruolo. Occorre, cioè, che nasca e si sviluppi un'indispensabile collaborazione tra pubblico e privato, che favorisca, nel tempo, il superamento degli elementi dissuasori interni ed esterni all'ente, di cui si è fatto cenno nei paragrafi 4 e 5 che precedono, che frenano, di fatto, la cooperazione di quest'ultimo nella reazione al fenomeno corruttivo intraorganizzativo.

In che modo? Lo precisano molto chiaramente le raccomandazioni del vertice G20 sulla lotta alla corruzione tenuto a Los Cabos (Messico) il 17 giugno 2012, ai cui lavori hanno partecipato, accanto alle organizzazioni OCSE, UNDOC, Transparency International, World Economic Forum (WEF) e International Chamber of Commerce (ICC), anche i rappresentanti del mondo industriale internazionale.

In particolare, sono state individuate e suggerite, tra le principali aree di intervento nella lotta alla corruzione, specifiche azioni da adottare, nel breve/medio periodo, da parte non solo del mondo delle imprese, ma anche delle autorità governative. Infatti, l'aspetto che merita di essere sottolineato è la rinnovata importanza riconosciuta, a livello internazionale, alla cooperazione tra autorità pubbliche ed enti privati e, soprattutto, all'esigenza di "facilitare" questa cooperazione istituendo specifici incentivi per l'ente che investa un ruolo attivo nella lotta alla corruzione e promuovendo programmi trasparenti e strutturati di "leniency" al fine di incoraggiare l'attività di self-reporting, vale a dire di segnalazione

28 SERENI, Andrea. Colpa e cooperazione investigativa nella responsabilità dell'ente da reato. Un confronto tra ordinamenti nella prospettiva della globalizzazione. Rivista trimestrale di diritto penale dell'economia, v. 3-4, p. 622623, 2018. 
spontanea di condotte illecite alle autorità competenti, e la cooperazione dell'ente stesso con l'autorità giudiziaria. Le raccomandazioni le seguenti.

«Government-driven actions - Government should:

Establish appropriate forms of "Hight Level Reporting Mechanisms" to address allegations of solicitation of bribes by public officials. In particular, governments should ensure the involvement of top authorities and set up such a mechanism in close cooperation with the support of the private sector and civil society.

Introduce clear and concrete system of leniency for companies that self-report corruption cases and/or cooperate in the investigation phase with relevant authorities.

Establish a framework for addressing multiple jurisdiction issues and effectively implement article 4.3 of the OECD Anti-Bribery Convention (on "Jurisdiction") and articles 48 and 49 of UNCAC (on "Law enforcement cooperation" and "Joint investigations").

Develop or revise relevant national rules, regulations, legislation or prosecutorial guidelines, as appropriate and permissible, to implement the principle of "Ne Bis In Idem", i) to take into account as a final judgment the closing of an anti-bribery investigation of a company in a foreign jurisdiction, including by means of a non-prosecution agreement, a deferred prosecution agreement, or a negotiated settlement, consent decree, or plea agreement, ii) to avoid duplicative penalties, sanctions and disgorgement of profit by different jurisdictions whether applied by criminal authorities or civil regulators» ${ }^{29}$.

\section{CONCLUSIONI PROVVISORIE.}

Il tema del ruolo da riconoscere all'ente nella lotta alla corruzione è vasto e in relazione a esso è possibile sviluppare diverse riflessioni.

In ambito internazionale, la cooperazione tra ente e autorità giudiziaria, innanzitutto nella gestione delle cc.dd. "red flags" rilevanti e

29 MANTOVANI, Massimo. Raccomandazioni del gruppo lavoro del B20 sulla anticorruzione e sulla trasparenza. Diritto del commercio internazionale, v. 3, p. $673-674,2012$. 
prima dell'instaurazione di formali procedimenti di indagine, è sempre più centrale. Dunque, si rafforza il convincimento che anche in Italia si debba avviare un percorso di cooperazione tra ente e autorità giudiziaria nella lotta alla corruzione serio e responsabile, libero dai pregiudizi e dalla sfiducia che spesso qualificano i due fronti, principalmente nella delicata fase delle indagini preliminari.

Un sistema della responsabilità dell'ente edificato sul condizionamento organizzativo di comportamenti collettivi non può eludere il problema di una «precisazione delle sfere di libertà e di autoresponsabilità individuali» ${ }^{30}$. È un problema anzitutto di valori, che chiama in causa la politica del diritto e, quindi, l'azione del legislatore.

Allora, occorre uno sforzo e un impegno legislativo per disciplinare, in modo rigoroso e credibile, e, per questa via, riuscire ad attivare un rapporto di fiducia biunivoco sulla volontà e sulla capacità dell'ente di prevenire e di ricercare condotte corruttive $\mathrm{o}$, più in generale, illecite, pure nella consapevolezza dei suoi ridotti poteri di indagine e nella capacità di cogliere le dinamiche interne al mondo delle imprese ${ }^{31}$.

$\grave{E}$ altrettanto noto che per attivare forme privatistiche di reale cooperazione occorrono incentivi seri, quali in parte vengono raccomandati dal G20 (sistemi di "leniency" per stimolare segnalazioni all'autorità giudiziaria di casi di corruzione o di segnali di allarme di corruzione tempestive, vale a dire prima dell'avvio di indagini da parte dell'organo

30 SERENI, Andrea. Colpa e cooperazione investigativa nella responsabilità dell'ente da reato. Un confronto tra ordinamenti nella prospettiva della globalizzazione. Rivista trimestrale di diritto penale dell'economia, v. 3-4, p. 628, 2018.

31 Come sottolinea SERENI, Andrea. Colpa e cooperazione investigativa nella responsabilità dell'ente da reato. Un confronto tra ordinamenti nella prospettiva della globalizzazione. Rivista trimestrale di diritto penale dell'economia, v. 3-4, p. 633, 2018, «il nuovo diritto "penale" delle società» non è «un diritto penale del singolo avvenimento, che accerta e reprime agitazioni in superficie, piccole increspature, "le onde che le maree sollevano col loro possente movimento"» (così BRAUDEL, Fernand. Scritti sulla storia. Milano: Bompiani, p. 11-12, 2016), ma «sembra costruito invece per catturare una storia di "lunga durata", delle relazioni strutturali e delle cause profonde che alimentano i singoli avvenimenti "rumorosi" colti dal diritto penale tradizionale»; v., anche, GENTILE, Emilio. Il fascino del persecutore: George L. Mosse e la catastrofe dell'uomo moderno. Roma: Carocci, p. 139-141, 2018. 
pubblico inquirente), e lo sforzo di individuare uno statuto comune di garanzie per l'ente cooperativo.

Tessendo le fila di complesse politiche della prevenzione e progettando un futuro ragionevolmente "mite" della responsabilità dell'ente, occorre, cioè, che il legislatore cerchi di definire un "modello" attorno cui rendere espliciti e certi i presupposti, i termini e i vantaggi della cooperazione dell'ente e precisare preventivamente la cornice della negoziazione con l'autorità giudiziaria, sì da garantire un equilibrato $\mathrm{e}$ accettabile bilanciamento tra efficienza preventivo-repressiva ed esigenze di libertà per l'ente e all'interno dell'ente.

Se il legislatore deciderà di compiere questo passo, esso potrà conseguire un risultato assai maggiore di quelli finora avuti: «l'adesione alla comune meta della prevenzione e la condivisione delle informazioni rilevanti» ${ }^{32}$. Soprattutto, si tratterà di un risultato, del quale vi è una reale e condivisa necessità, che muove nel solco auspicato dalle convenzioni internazionali e dalle raccomandazioni del G20.

Anche a costo di apparire superati dai tempi e distonici rispetto al clima culturale della post-modernità ${ }^{33}$, si è, tutt'ora, convinti che «la Costituzione sia in grado di fornire un contributo fondamentale alla promozione di un diritto penale razionale e rispettoso di standard di civiltà» ${ }^{34}$. In particolare, e in conclusione, il progetto di enunciazione di un "manifesto" della cooperazione tra ente e autorità giudiziaria nella lotta alla corruzione dovrebbe muovere dalla rinnovata consapevolezza - in capo, anzitutto, al legislatore, ma, a seguire, all'ente e alla società civile tutta - che il «fine ultimo dell'organizzazione sociale» risiede, in forza dell'art. 2 Cost., nello «sviluppo di ogni singola persona umana» ${ }^{35}$. Ciò significa che «non è l'uomo in funzione dello Stato, ma quest'ultimo in

32 CENTONZE, Francesco. Responsabilità da reato degli enti e agency problems. I limiti del d.lgs.vo n. 231 del 2001 e le prospettive di riforma. Rivista italiana di diritto e procedura penale, v. 3, p. 986, 2017.

33 BAUMAN, Zygmunt. Il disagio della postmodernità. Milano: Editori Laterza, 2018.

34 DOLCINI, Emilio. Pena e Costituzione. Rivista italiana di diritto e procedura penale, v. 1, p. 29, 2019.

35 Corte cost., 29 aprile 1999 (dep. 10 maggio 1999), n. 167, in Giurisprudenza italiana, 2000, p. 683. 
funzione dell'uomo ${ }^{36}$. Sull'inviolabilità dei principi supremi - «che non possono essere sovvertiti o modificati nel loro contenuto essenziale» ${ }^{37}$, che acquistano il senso classico di «garanzia negativa contro indebite intromissioni dell'autorità $[\ldots]$ nella sfera riservata all'individuo» ${ }^{38}$, che valgono non solo nei confronti dei poteri pubblici ma, secondo il principio della Drittwirkung, anche nei rapporti interprivati ${ }^{39}$ e la cui tutela si estende, sempre in forza dell'art. 2 Cost., alle «formazioni sociali ove si svolge la personalità» umana, ivi compreso l'ente - dovrebbe, dunque, svilupparsi questo "manifesto" dei rapporti cooperativi tra l'ente e l'autorità giudiziaria. Vale a dire sulla consapevolezza che l'ente deve essere riconosciuto e garantito in quanto «consenta e favorisca il libero sviluppo della persona» e «garantisca la tutela di "interessi diffusi" rilevanti costituzionalmente $»^{40}$.

\section{BIBLIOGRAFIA}

AMARELLI, Giuseppe. L'attenuante della dissociazione attuosa (art. 8 d.l. n. 152/1991). In: MAIELLO, Vincenzo (a cura di). La legislazione penale in materia di criminalità organizzata, misure di prevenzione e armi. Torino: Giappichelli, 2015. p. $197-238$.

36 MORTATI, Costantino. Istituzioni di diritto pubblico. Padova: Cedam, p. 155, 1975.

37 Corte cost., 15 dicembre 1988 (dep. 29 dicembre 1988), n. 1146, in Foro italiano, v. 1, p. 609, 1989.

38 ONIDA, Valerio. Relazione. In: AA. VV. (a cura di). I diritti fondamentali oggi: atti del V convegno dell'associazione italiana dei costituzionalisti. Padova: Cedam, 1995. p. 69.

39 Corte cost., 2 aprile 1969 (dep. 17 aprile 1969), n. 84, in DeJure; Corte cost., 12 luglio 1979 (dep. 26 luglio 1979), n. 88, in Giurisprudenza costituzionale, v. 1, p. 656, 1979; Corte cost., 30 giugno 1986 (dep. 14 luglio 1986), n. 184, in Giurisprudenza costituzionale, v. 8, 1986; Corte cost., 6 febbraio 2006 (dep. 10 febbraio 2006), n. 50, in Giurisprudenza italiana, v. 12, p. 2242, 2006; Corte cost., 21 giugno 2006 (dep. 6 luglio 2006), n. 266, in Il civilista, v. 4, p. 13, 2009; Corte cost., 11 luglio 2007 (dep. 24 luglio 2007), n. 321, in Giurisprudenza costituzionale, v. 4, p. 3124, 2007.

40 BARBERA, Augusto. Sub art. 2 Cost. In: BRANCA, Giuseppe (a cura di). Commentario della Costituzione. Roma: Società Editrice del Foro Italiano, 1975. p. 109. 
ARLEN, Jennifer. L'applicazione della legge penal-societaria negli Stati Uniti: l'uso delle transazioni per trasformare imprese potenzialmente criminali in tutori dell'ordine. Rivista trimestrale di diritto penale dell'economia, v. 1-2, p. 1-40, 2018. BAUMAN, Zygmunt. Modernità liquida. Roma-Bari: Editori Laterza, 2011.

BAUMAN, Zygmunt. Il disagio della postmodernità. Milano: Editori Laterza, 2018. BERTOLINO, Marta. Dall'organizzazione all'individuo: crimine economico e personalità, una relazione da scoprire. Criminalia, p. 15-35, 2014.

BIANCHETTI, Raffaele. La paura del crimine: un'indagine criminologica in tema di mass media e politica criminale ai tempi dell'insicurezza. Milano: Giuffrè, 2018.

BONELLI, Franco. "Collaborazione" tra autorità pubbliche e società private per un'efficace lotta alla corruzione. Diritto del commercio internazionale, v. 3, p. 675-687, 2012.

BRAUDEL, Fernand. Scritti sulla storia. Milano: Bompiani, 2016.

CARRERA, Francesco. Programma del corso di diritto criminale: parte generale. Lucca: Il Mulino, 1871.

CENTONZE, Francesco. Responsabilità da reato degli enti e agency problems. I limiti del d.lgs.vo n. 231 del 2001 e le prospettive di riforma. Rivista italiana di diritto e procedura penale, v. 3, p. 945-987, 2017.

DI MARTINO, Alberto. La sequenza infranta: i profili della dissociazione tra reato e pena. Milano: Giuffrè, 1998.

DOLCINI, Emilio. Pena e Costituzione. Rivista italiana di diritto e procedura penale, v. 1, p. 3-33, 2019.

FILANGIERI, Gaetano. La Scienza della Legislazione. Napoli: Grimaldi \& C., 2003. FIORELLA, Antonio. Logica, criticità e riforme del sistema punitivo per l'illecito dell'ente da reato. Rivista italiana di diritto e procedura penale, v. 1, p. 517-531, 2019.

FORTI, Gabrio. L'immane concretezza: metamorfosi del crimine e controllo penale. Milano: Raffaello Cortina Editore, 2000.

FOUCAULT, Paul-Michel. La società punitiva: corso al Collège de France (19721973). Milano: Feltrinelli, 2016.

FOUCAULT, Paul-Michel. Sorvegliare e punire: nascita della prigione. Torino: Einaudi, 1993. 
GARAPON, Antoine. La responsabilità delle persone giuridiche e le nuove "regole del gioco" mondiale. I paradigmatici casi BNP-Paribas e Alstom. In: CENTONZE, Francesco-MANTOVANI, Massimo (a cura di). La responsabilità "penale" degli enti: dieci proposte di riforma. Bologna: Il Mulino, 2016. p. 56-100.

GENTILE, Emilio. Il fascino del persecutore: George L. Mosse e la catastrofe dell'uomo moderno. Roma: Carocci, 2018.

GRECO, Francesco. Perché "fallisce" il sistema dei controlli? In: BRESCIA, FrancoTORCHIA, Luisa-ZOPPINI, Andrea (a cura di). Metamorfosi del diritto delle società?: seminario per gli ottant'anni di Guido Rossi. Napoli: Editoriale Scientifica, 2012.

HASSEMER, Winfried. Perché punire è necessario. Bologna: Il Mulino, 2012.

MAIELLO, Vincenzo. Fuga dalla sanzione e postmodernità penalistica. In: MOCCIA, Sergio (a cura di). La giustizia contrattata: dalla bottega al mercato globale. Napoli: Edizioni Scientifiche Italiane, 1998. p. 115 ss.

MANES, Vittorio. L'estensione dell'art. 4-bis ord. pen. ai delitti contro la P.A.: profili di illegittimità costituzionale. Rivista trimestrale di diritto penale contemporaneo, v. 2, p. 105-122, 2019.

MANTOVANI, Massimo. Raccomandazioni del gruppo lavoro del B20 sulla anticorruzione e sulla trasparenza. Diritto del commercio internazionale, v. 3, p. 671-674, 2012.

MASULLO, Maria Novella. L'emersione del patto corruttivo: il nuovo fronte degli strumenti premiali e investigativi. Rivista italiana di diritto e procedura penale, $\mathrm{v}$. 3, p. $1257-1287,2019$.

MAZZACUVA, Federico. La diversione per gli enti collettivi nell'esperienza anglo-americana. Alcuni spunti de iure condendo. Rivista trimestrale di diritto penale contemporaneo, v. 2, p. 80-88, 2016.

MOCCIA, Sergio. La "promessa non mantenuta": ruolo e prospettive del principio di determinatezza/tassatività nel sistema penale italiano. Napoli: Edizioni Scientifiche Italiane, 2001.

MORTATI, Costantino. Istituzioni di diritto pubblico. Padova: Cedam, 1975.

ONIDA, Valerio. Relazione. In: AA. VV. (a cura di). I diritti fondamentali oggi: atti del V convegno dell'associazione italiana dei costituzionalisti. Padova: Cedam, 1995. p. 69 ss. 
PADOVANI, Tullio. Il traffico delle indulgenze. "Premio" e "corrispettivo" nella dinamica della punibilità. Rivista italiana di diritto e procedura penale, v. 2 , p. 398-436, 1986.

PALIERO, Carlo Enrico. Criminalità economica e criminalità organizzata: due paradigmi a confronto. In: BARILLARO, Michele (a cura di). Criminalità organizzata e sfruttamento delle risorse territoriali. Milano: Giuffrè, 2004. p. 141 ss.

PATRONO, Paolo. Diritto penale dell'impresa e interessi umani fondamentali. Padova: Cedam, 1993.

PELLISSERO, Marco. La nuova disciplina della corruzione tra repressione e prevenzione. In: MATTARELLA, Bernardo Giorgio-PELLISSERO, Marco (a cura di). La legge anticorruzione. Torino: Giappichelli, 2013. p. 347-353.

PIERGALLINI, Carlo. Premialità e non punibilità nel sistema della responsabilità da reato degli enti. Diritto penale e processo, v. 4, p. 530-549, 2019.

PISTORELLI, Luca. La responsabilità da reato degli enti: un bilancio applicativo. Rivista trimestrale di diritto penale dell'economia, v. 3-4, p. 610-622, 2017.

PULITANÒ, Domenico. Le cause di non punibilità dell'autore della corruzione e dell'infiltrato e la riforma dell'art. 4-bis. Diritto penale e processo, v. 5, p. 600607, 2019.

RACITI, Annamaria. Il criminale economico nella ricerca criminologica: dall'opera di Sutherland alle più recenti formulazioni teoretiche. Rivista trimestrale di diritto penale dell'economia, v. 3, p. 677-699, 2005.

RUGA RIVA, Carlo. Il premio per la collaborazione processuale. Milano: Giuffrè, 2002.

RUGGIERO, Rosa Anna. Non prosecution agreement e criminalità d'impresa negli U.S.A.: il paradosso del liberismo economico. Diritto penale contemporaneo, p. 1-18, 12 ottobre 2015.

SANTORIELLO, Ciro. Anche nei confronti degli enti collettivi il decreto anticorruzione mostra inutili e incoerenti "furori sanzionatori". Diritto penale $e$ processo, v. 6, p. 756-761, 2019.

SERENI, Andrea. Colpa e cooperazione investigativa nella responsabilità dell'ente da reato. Un confronto tra ordinamenti nella prospettiva della globalizzazione. Rivista trimestrale di diritto penale dell'economia, v. 3-4, p. 620-642, 2018.

SERENI, Andrea. L'ente guardiano: l'autorganizzazione del controllo penale. Torino: Giappichelli, 2016. 
SUTHERLAND, Edwin Hardin. White Collar Criminality. American Sociological Review, v. 5, n. 1, p. 1-12, 1940. https://doi.org/10.2307/2083937

SUTHERLAND, Edwin Hardin. White Collar Crime: The Uncut Version. New Heaven: Yale University Press, 1983.

VANNUCCI, Alberto. La corruzione nel sistema politico italiano a dieci anni da "mani pulite". In: FORTI, Gabrio (a cura di). Il prezzo della tangente: la corruzione come sistema a dieci anni da "mani pulite". Milano: Vita e Pensiero, 2003. p. 1-68.

\section{Additional information and author's declarations (scientific integrity)}

Conflict of interest declaration: the authors confirm that there are no conflicts of interest in conducting this research and writing this article.

Declaration of authorship: all and only researchers who comply the authorship requirements of this article are listed as authors; all coauthors are fully responsible for this work in its entirety.

- Riccardo Roscini-Vitali: conceptualization, methodology, data curation, investigation, writing - original draft, validation, writing - review and editing, final version approval.

- Vittore d'Acquarone: conceptualization, methodology, data curation, investigation, writing - original draft, validation, writing - review and editing, final version approval.

Declaration of originality: the authors assure that the text here published has not been previously published in any other resource and that future republication will only take place with the express indication of the reference of this original publication; they also attest that there is no third-party plagiarism or self-plagiarism. 
Dados do processo editorial

(http://www.ibraspp.com.br/revista/index.php/RBDPP/about/editorialPolicies)

- Recebido em: 07/08/2020

Equipe editorial envolvida

- Controle preliminar e verificação de plágio:

- Editor-chefe: 1 (VGV) 15/08/2020

- Avaliação 1:03/09/2020

- Editor-associado: 1 (RO)

- Avaliação 2: 07/09/2020

- Revisores: 2

- Decisão editorial preliminar: 20/09/2020

- Retorno rodada de correções: 30/09/2020

- Decisão editorial final: 01/10/2020

\section{COMO CITAR ESTE ARTIGO:}

ROSCINI-VITALI, Riccardo; D'ACQUARONE, Vittore. Sul ruolo dell'ente nel contrasto alla corruzione: un moderno Atlante. Revista Brasileira de Direito Processual Penal, Porto Alegre, vol. 6, n. 3, p. 1389-1413, set./dez. 2020. https://doi.org/10.22197/rbdpp.v6i3.440

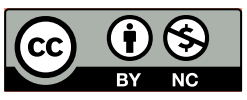

Esta obra está licenciada com uma Licença Creative Commons Atribuição-NãoComercial 4.0 Internacional. 